\title{
A Note on Translation and Transliteration
}

Except where I have indicated otherwise, all translations are my own. In some cases I have given my own translation but have cited a published English translation. I have indicated this in the notes.

For Russian terms and for titles of books and articles I have used the international scholarly transliteration style favored in Slavic studies. For personal names I have either used the Library of Congress system or given the form that is likely to be most familiar to English speakers. 
